\title{
Determination of Injection Site in Flexor Digitorum Longus for Effective and Safe Botulinum Toxin Injection
}

\author{
Hong Geum Kim, MD, Myung Eun Chung, MD, PhD, Dae Heon Song, MD, PhD, \\ Ju Yong Kim, MD, Bo Mi Sul, MD, Chang Hoon Oh, MD, Nam Su Park, MD
}

Department of Rehabilitation Medicine, St. Paul's Hospital, The Catholic University of Korea College of Medicine, Seoul, Korea

Objective To determine the optimal injection site in the flexor digitorum longus (FDL) muscle for effective botulinum toxin injection.

Methods Fourteen specimens from eight adult Korean cadavers were used in this study. The most proximal medial point of the tibia plateau was defined as the proximal reference point; the most distal tip of the medial malleolus was defined as the distal reference point. The distance of a line connecting the proximal and distal reference points was defined as the reference length. The X-coordinate was the distance from the proximal reference point to the intramuscular motor endpoint (IME), or motor entry point (MEP) on the reference line, and the Y-coordinate was the distance from the nearest point from MEP on the medial border of the tibia to the MEP. IME and MEP distances from the proximal reference point were evaluated using the raw value and the $\mathrm{X}$-coordinate to reference length ratio was determined as a percentage.

Results The majority of IMEs were located within $30 \%-60 \%$ of the reference length from the proximal reference point. The majority of the MEPs were located within $40 \%-60 \%$ of the reference length from the proximal reference point.

Conclusion We recommend the anatomical site for a botulinum toxin injection in the FDL to be within a region $30 \%-60 \%$ of the reference length from the proximal reference point.

Keywords Flexor digitorum longus, Botulinum toxins, Spasticity, Claw toes

Received May 29, 2014; Accepted September 2, 2014

Corresponding author: Nam Su Park

Department of Rehabilitation Medicine, St. Paul's Hospital, The Catholic University of Korea College of Medicine, 180 Wangsan-ro, Dongdaemungu, Seoul 130-709, Korea

Tel: +82-2-957-2307, Fax: +82-2-968-2307, E-mail: park9262@hotmail.com

@ This is an open-access article distributed under the terms of the Creative Commons Attribution Non-Commercial License (http://creativecommons. org/licenses/by-nc/3.0) which permits unrestricted noncommercial use, distribution, and reproduction in any medium, provided the original work is properly cited.

Copyright $\odot 2015$ by Korean Academy of Rehabilitation Medicine

\section{INTRODUCTION}

Spasticity is a motor disorder characterized by a velocity-dependent increase in tonic stretch reflexes, resulting from hyperexcitability of the stretch reflex, which is a component of upper motor neuron syndrome [1]. Various insults, such as stroke, traumatic brain injury, spinal cord injury, cerebral palsy, and multiple sclerosis, can cause spasticity. Lower limb spasticity is related with equinus, varus, and clawing toes; the condition results in 
poor balance and reduced gait capacity [2]. Clawing toes can interfere with distal positioning and ambulatory capacity; the condition ultimately results in a limitation of activities required for daily living (ADL). The spastic foot pain and skin breakdown that results from toe clawing can significantly impair quality of life [3-6].

Several studies have identified the effectiveness and safety of treatment following botulinum toxin injection $[4,6-8]$. Clinically, toe clawing is treated via injection of the long toe flexor with botulinum toxin [6,9]. Some studies have shown that botulinum toxin injections for flexor digitorum longus (FDL) improve the spasticity, ambulatory capacity, and functional ability in patients with toe clawing $[4,6]$. Botulinum toxin injection for FDL in patients with toes clawing can ameliorate spastic foot pain [6].

Botulinum toxin reversibly reduces muscle tone. Because botulinum toxin inhibits the release of acetylcholine by the presynaptic membrane of the motor endplate, the injection should be administered into the motor endplate zone [10]. The motor endplate zone is where motor endplates are the densest; several studies have proposed that the regions where intramuscular nerve endings are the most distributed are consistent with the motor endplate zone $[11,12]$. Numerous studies have explored the optimal site for botulinum toxin injection in the management of lower limb spasticity. The motor entry point (MEP) was defined as the location where the motor nerve penetrated the muscle belly; the intramuscular motor endpoint (IME) was defined as the location where the intramuscular motor nerve ended. Injection sites evaluated included the MEP and distribution of IME of the triceps surae [13,14], hamstring muscle [15], the psoas muscle [16], and tibialis posterior muscle [17]. In one humanbased study, endplate-targeted botulinum toxin injection was more effective than non-targeted injection in spastic biceps [18]. Therefore, in order to properly administer toxin injection for FDL, precise knowledge of the appropriate anatomical injection site to the target muscles is indispensable $[13,19]$. However, the extant literature has not yet determined the optimal anatomical site for an effective botulinum toxin injection of FDL. The aim of the present study is to determine the optimal anatomical site and motor endplate zone for botulinum toxin injection to manage spasticity of FDL. In this study, we investigated the IME and MEP of the FDL for an effective and safe botulinum toxin injection.

\section{MATERIALS AND METHODS}

Fourteen specimens from eight adult Korean cadavers ( 5 males and 3 females, age ranging from 52 to 79 years) were used in this study. Specimens with severe deformities, such as leg contractures, equinovarus, or equinovalgus, or a history of trauma or amputation of the legs, were excluded from the study. An incision was made from the medial knee to the malleolus of the tibia; the posteromedial skin of the calf was removed. The gastrocnemius and soleus muscle were then removed from the tibia bone. This allowed for the FDL to be exposed and identified, as well as the tibial nerve descending from the popliteal fossa and the branching nerves. We traced the branching nerve that innervated the FDL.

The MEP was defined as the location where the motor nerve penetrated the muscle belly (Fig. 1). The muscle was cut along the nerves to investigate the intramuscular course of the nerves. We held onto the nerves with just enough tension as to not hurt them; then, we continued dissecting the musculature surrounding the nerves with blunt forceps. The IME was defined as the location where the intramuscular motor nerve ended, which was determined by observation with the use of magnifying glasses (Fig. 1). Two bony landmarks were selected as reference points: the most proximal medial point of the tibia plateau was defined as the proximal reference point, and the most distal tip of the medial malleolus was defined as the distal reference point. The line connecting the proximal and distal reference points was defined as the reference line. The length of reference line was defined as the reference length. The X-coordinate was the distance from the proximal reference point to the MEP or IME on

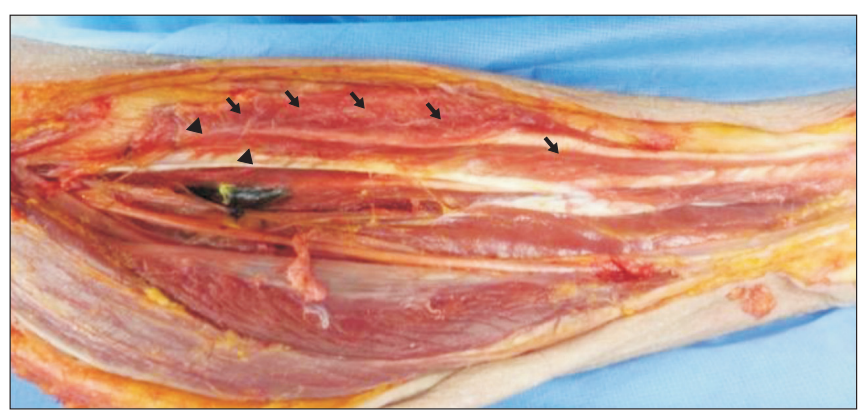

Fig. 1. Photographs of the posteromedial aspect of the leg showing the motor entry point (arrowhead) and intramuscular motor endpoint (arrow). 
the reference line, and the Y-coordinate was the distance from the medial border of the tibia to the MEP on a line perpendicular to the reference line (Fig. 2). The distances of MEP and IME from the proximal reference point were evaluated using the raw value, and the $\mathrm{X}$-coordinate to reference length ratio was determined as a percentage (Fig. 2).

\section{RESULT}

The mean value of the reference line for the 14 specimens was $333.96 \pm 25.20 \mathrm{~mm}$. There were a total of 29 MEPs in the 14 specimens examined, with 1 MEP in 1 specimen, 2 MEPs in 11 specimens, and 3 MEPs in 2 specimens.

\section{Location of IMEs}

The mean distance from the proximal reference point to the most proximal IME and the ratio of the distance to the reference length on X-coordinate were $117.64 \pm 13.24$ $\mathrm{mm}$ and $35.00 \% \pm 3.72 \%$, respectively. The mean distance from the proximal reference point to the most distal IME and the ratio of the distance to the reference length on $\mathrm{X}$-coordinate were $264.64 \pm 25.90 \mathrm{~mm}$ and $78.57 \% \pm 4.15 \%$, respectively. The majority of IMEs were located within $30 \%-60 \%$ of the reference length from the proximal reference point (Table 1).

\section{Location of MEPs}

The mean distance of the MEP from the proximal reference point and the ratio of the distance to the reference length were $158.03 \pm 37.39 \mathrm{~mm}$ and $46.78 \% \pm 9.65 \%$,

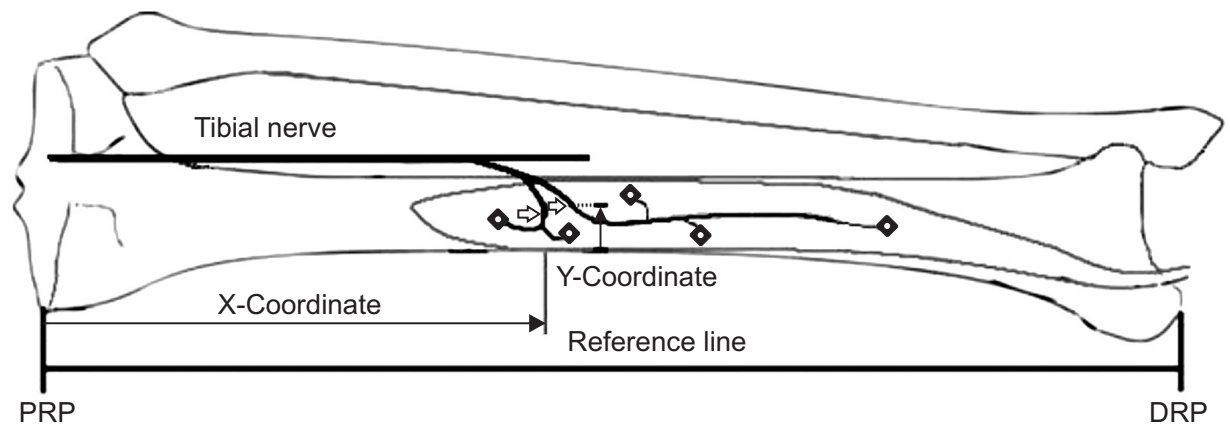

Fig. 2. The X-coordinate was the distance from the proximal reference point (PRP) to the motor entry point (arrow) or intramuscular motor endpoint (rhombus) on the reference line, and the Ycoordinate was the distance from the medial border of the tibia to the motor entry point on a line perpendicular to the reference line. DRP, distal reference point.

Table 1. Distance of the intramuscular motor endpoint (IME) of flexor digitorum longus muscle from the proximal reference point

\begin{tabular}{lcccccc}
\hline & \multicolumn{5}{c}{ Distance from the proximal reference point (ratio to the reference length) } \\
\cline { 2 - 7 } & $\mathbf{3 0 \% - 4 0 \%}$ & $\mathbf{4 0 \% - 5 0 \%}$ & $\mathbf{5 0 \% - 6 0 \%}$ & $\mathbf{6 0 \% - 7 0 \%}$ & $\mathbf{7 0 \% - 8 0 \%}$ & $\mathbf{8 0 \% - 9 0 \%}$ \\
\hline The number of IME & $15(24.59)$ & $18(29.51)$ & $13(21.31)$ & $6(9.84)$ & $9(14.75)$ & $6(9.84)$ \\
Average \pm SD & $117.07 \pm 11.47$ & $146.94 \pm 15.33$ & $183.46 \pm 17.16$ & $221.67 \pm 15.71$ & $247.78 \pm 16.03$ & $283.33 \pm 26.39$ \\
& $(34.75 \pm 3.00)$ & $(43.81 \pm 3.22)$ & $(54.46 \pm 2.87)$ & $(65.39 \pm 2.91)$ & $(76.03 \pm 2.89)$ & $(81.87 \pm 1.54)$ \\
\hline
\end{tabular}

Table 2. Distance of the motor entry point (MEP) of the flexor digitorum longus muscle from the proximal reference point

\begin{tabular}{|lcccc}
\hline & \multicolumn{4}{c}{ Distance from the proximal reference point (ratio to the reference length) } \\
\cline { 2 - 5 } & $\mathbf{3 0 \% - 4 0 \%}$ & $\mathbf{4 0 \% - 5 0 \%}$ & $\mathbf{5 0 \% - 6 0 \%}$ & $\mathbf{6 0 \% - 7 0 \%}$ \\
\hline The number of MEP & $6(20.69)$ & $12(41.38)$ & $9(31.03)$ & $2(6.90)$ \\
Average \pm SD & $119.83 \pm 13.63$ & $142.0 \pm 13.68$ & $185.56 \pm 15.53$ & $245.0 \pm 21.21$ \\
& $(35.22 \pm 2.14)$ & $(43.07 \pm 3.60)$ & $(55.34 \pm 3.46)$ & $(65.33 \pm 5.66)$ \\
Y-coordinate & & & & \\
Average \pm SD & $19.5 \pm 2.26$ & $17.9 \pm 2.91$ & $16.67 \pm 3.04$ & $17.5 \pm 0.71$ \\
\hline Minimum distance & 16 & 12 & 10 & 17 \\
\hline Maximum distance & 22 & 22 & 20 & 18 \\
\hline
\end{tabular}


respectively. The distance from the proximal reference point to the most proximal MEP and the ratio of the distance to the reference length were $107 \mathrm{~mm}$ and $32.42 \%$, respectively. The distance from the most distal MEP to the proximal reference point and the ratio of the distance to the reference length on the X-coordinate were $260 \mathrm{~mm}$ and $69.33 \%$, respectively. The majority of the MEPs were located within $40 \%-60 \%$ of the reference length from the proximal reference point. The mean distance of the MEP from the medial border of the tibia on the Y-coordinate was $17.83 \pm 2.82 \mathrm{~mm}$ (Table 2).

\section{DISCUSSION}

This is to our knowledge the first study to demonstrate the optimal injection site in FDL and to relate it to external bony landmark for effective and safe botulinum toxin injection. The IMEs of FDL are distributed along the longitudinal muscle belly. The majority of MEPs of the FDL were found within a $40 \%-60 \%$ region of the reference length from the proximal reference point; the majority of the IMEs of the FDL were found within a $30 \%-60 \%$ region of the reference length from the proximal reference point. From the results of this study, we can propose the optimal injection site for botulinum toxin injection in FDL.

There are numerous studies to localize the optimal site for botulinum injection. Some studies have defined the location for optimal site of botulinum toxin injection as the site where the motor nerve first penetrates the muscle belly, which is defined as the MEP in this study $[14,20]$. However, other studies indicated that botulinum injections at the motor endplate zone result in more effective paralysis with a smaller dose administered $[21,22]$. The motor endplate zone is the region where motor endplates in muscle are highly concentrated. Current knowledge on the localization of the motor endplate zone is primary based on a few, older histological studies. In animal and human muscles, the motor endplates are not randomly distributed [10]. As a general rule, the motor endplates are equidistant from the poles of their respective muscle fibers [13]. Also, the distribution of motor endplates varies according to the type of muscle, its function, and structure and motor endplates concentration in specific region, which is the motor endplate zone [13]. For example, the motor endplate zone, in unipennate muscles except in the gracilis and sartorius forms a transverse band through the middle of the muscle [23,24]. In bipennate converging muscles (e.g., gastrocnemius), the motor endplate zone forms an upwardly convex shape [24]. Some studies indicated that the region where the motor nerve terminal endings are dense is consistent with the motor endplate zone revealed by acetylcholinesterase (AChE) staining [25,26]. Several studies proposed that the regions where intramuscular nerve endings are distributed the most are the zones where motor endplates are the densest, which is the motor endplate zone $[11,12]$. Recent several studies using anatomical microdissection evaluated the intramuscular motor nerve endings to localize the optimal injection site for effective and safe botulinum injection in relation to other external references $[13,15$ 17,27-30]. Parratte et al. demonstrated that optimal injection sites are three-quarters of the way up the leg for the medial head of the gastrocnemius and four-fifths of the way up the leg for the lateral head of the gastrocnemius [13]. Injection of the tibialis posterior muscle at about the distal limit of the proximal third of the line between the head of the fibula and the intermalleolar axis seems optimal $[31,32]$. The optimal injection site for the semitendinosus muscle and the semimembranosus muscle seem to be between $21 \%$ and $50 \%$ and between $64 \%$ and $77 \%$ of the reference line from the ischial tuberosity to the lateral femoral condyle, respectively [31,33]. These studies demonstrated optimal site for botulinum toxin injection by localization of region where motor nerve endings are distributed the most. Our study evaluated distribution of motor nerve endings in FDL with anatomical microdissection and can indicate optimal injection zone for botulinum toxin injection in FDL. In accordance with these studies, the majority of IMEs in FDL are concentrated in specific region located within $30 \%-60 \%$ of the reference length from the proximal reference point, which is motor endplate zone.

Botulinum toxin primarily inhibits the release of acetylcholine from the motor endplate of the neuromuscular junction; spasticity is improved by the decrease of tension and spasm in the target muscle. The toxin injection needs to be localized to the motor endplate in order to be effective botulinum toxin injection [21,22]. Histological studies in rabbit models showed that injections only $5 \mathrm{~mm}$ away from an endplate result in a $50 \%$ decrease in toxin denervation and paralysis. The studies also demonstrated that injections directly into motor endplates show 
the greatest level of paralysis for a given dose of botulinum toxin [21]. One human study demonstrated that endplate-targeted botulinum toxin injection is more effective than non-targeted injection in spastic biceps [18].

Botulinum toxin is expensive, and it can cause some side effects of paralysis due to diffusion to adjacent muscles; therefore, a minimum amount of botulinum toxin, adequate to give the maximum therapeutic outcome, must be injected at the motor endplate. Botulinum toxin easily passes through muscle fascia, even at subclinical doses and fascia reduces the spread by only $23 \%$ [34]. Therefore, the spread of botulinum toxin can be prevented only by delivering small doses to the motor endplate zone of a target muscle [31]. The botulinum toxin dose per muscle with precise injection can also be minimized [34]. Consequently, identifying the optimal and safe injection site for the target muscle is critical. To overcome the issues cited above, proper localization of botulinum toxin injection site for each muscle is necessary. Thus, our finding of the ideal botulinum toxin injection site of FDL makes effective and safe botulinum toxin injection for FDL possible.

Based on our findings, we recommend the clinically optimum site for a botulinum toxin injection for the FDL to be within a region $30 \%-60 \%$ of the reference length from the proximal reference point.

There are some limitations to this study's findings. First, the number (sample size) of specimens used in this study was relatively small. However, the present study is still the first to investigate the anatomical IME of FDL; thus, this study can be seen as a starting point for further studies using a larger sample size. Second, the study's findings cannot be directly applied to children. Indeed, botulinum toxin injections can be administered in children who have neuromusculoskeletal disorders, such as cerebral palsy in order to improve ambulatory ability and manage pain. Although this study localizes the injection site by means of percentage of a reference length, this indirectly allows the result to be applied to children. However, a proper study comparing the localization of motor endplates in children and adults has not been conducted. One animal study has documented that the distribution of neuromuscular junction in juvenile and adult rats is similar [35]. Therefore, further studies involving children will be required for the findings to be generalized to that population. Third, although we traced the motor nerve until it was not visible, the IME in the present study does not correspond to motor endplate strictly. The endpoint of motor nerve branches is localized by tracing the nerves with microdissection to their terminal branches or by serially sectioning the muscle histologically [36]. However, dissecting the most terminal branches to the level of the motor endplate is almost impossible [36]. Histological studies include serially staining every section of the muscle for nerves, and then tracing and reconstructing the nerve branches from each section [36]. However, because of tissue collection and staining, this method may prevent reconstruction of the nerve branches. The use of Sihler's stain can overcome these problems [37,38]. Nerve branches in muscle can be identified in their original 3-dimensional model. However, the very end of the nerve branches might still not be definitely identified, so tracing the level to the motor endplate is difficult [36]. An anatomical study with a histochemical approach can be alternative to localize motor endplates. AChE staining is a sensitive method to localize motor endplates and specific method in combination with an anatomical dissection identifying motor nerve endings [36]. Therefore, further study with AChE staining to localize motor endplate zone for botulinum toxin injection of FDL will be required.

In summary, this is the first cadaveric study to evaluate the anatomical location of IME and MEP of the FDL. We believe that findings of this study will be useful in determining a more effective and safe botulinum toxin injection in the FDL.

\section{CONFLICT OF INTEREST}

No potential conflict of interest relevant to this article was reported.

\section{REFERENCES}

1. Assessment: the clinical usefulness of botulinum toxin-A in treating neurologic disorders. Report of the Therapeutics and Technology Assessment Subcommittee of the American Academy of Neurology. Neurology. 1990;40:1332-6.

2. Rousseaux M, Compere S, Launay MJ, Kozlowski O. Variability and predictability of functional efficacy of botulinum toxin injection in leg spastic muscles. J Neurol Sci 2005;232:51-7. 
3. Bakheit AM. The pharmacological management of post-stroke muscle spasticity. Drugs Aging 2012; 29:941-7.

4. Lim EC, Ong BK, Seet RC. Botulinum toxin-A injections for spastic toe clawing. Parkinsonism Relat Disord 2006;12:43-7.

5. Thibaut A, Chatelle C, Ziegler E, Bruno MA, Laureys S, Gosseries O. Spasticity after stroke: physiology, assessment and treatment. Brain Inj 2013;27:1093-105.

6. Suputtitada A. Local botulinum toxin type A injections in the treatment of spastic toes. Am J Phys Med Rehabil 2002;81:770-5.

7. Hesse S, Brandi-Hesse B, Bardeleben A, Werner C, Funk M. Botulinum toxin A treatment of adult upper and lower limb spasticity. Drugs Aging 2001;18:25562.

8. Burbaud P, Wiart L, Dubos JL, Gaujard E, Debelleix X, Joseph PA, et al. A randomised, double blind, placebo controlled trial of botulinum toxin in the treatment of spastic foot in hemiparetic patients. J Neurol Neurosurg Psychiatry 1996;61:265-9.

9. Yelnik AP, Bonan IV. Post stroke hemiplegia: lower limb benefit from botulinum toxin (review). Ann Readapt Med Phys 2003;46:281-5.

10. Childers MK. Targeting the neuromuscular junction in skeletal muscles. Am J Phys Med Rehabil 2004;83(10 Suppl):S38-44.

11. Hwang K, Jin S, Hwang SH, Lee KM, Han SH. Location of nerve entry points of flexor digitorum profundus. Surg Radiol Anat 2007;29:617-21.

12. Lepage D, Parratte B, Tatu L, Vuiller F, Monnier G. Extra- and intramuscular nerve supply of the muscles of the anterior antebrachial compartment: applications for selective neurotomy and for botulinum toxin injection. Surg Radiol Anat 2005;27:420-30.

13. Parratte B, Tatu L, Vuillier F, Diop M, Monnier G. Intramuscular distribution of nerves in the human triceps surae muscle: anatomical bases for treatment of spastic drop foot with botulinum toxin. Surg Radiol Anat 2002;24:91-6.

14. Kim HS, Hwang JH, Lee PK, Kwon JY, Oh-Park MY, Kim JM, et al. Localization of the motor nerve branches and motor points of the triceps surae muscles in Korean cadavers. Am J Phys Med Rehabil 2002;81:765-9.

15. An XC, Lee JH, Im S, Lee MS, Hwang K, Kim HW, et al. Anatomic localization of motor entry points and in- tramuscular nerve endings in the hamstring muscles. Surg Radiol Anat 2010;32:529-37.

16. Van Campenhout A, Hubens G, Fagard K, Molenaers G. Localization of motor nerve branches of the human psoas muscle. Muscle Nerve 2010;42:202-7.

17. Lee JH, Lee BN, An X, Chung RH, Han SH. Location of the motor entry point and intramuscular motor point of the tibialis posterior muscle: for effective motor point block. Clin Anat 2011;24:91-6.

18. Gracies JM, Lugassy M, Weisz DJ, Vecchio M, Flanagan S, Simpson DM. Botulinum toxin dilution and endplate targeting in spasticity: a double-blind controlled study. Arch Phys Med Rehabil 2009;90:9-16.e2.

19. O'Brien CF. Injection techniques for botulinum toxin using electromyography and electrical stimulation. Muscle Nerve Suppl 1997;6:S176-80.

20. Crystal R, Malone AA, Eastwood DM. Motor points for neuromuscular blockade of the adductor muscle group. Clin Orthop Relat Res 2005;437:196-200.

21. Shaari CM, Sanders I. Quantifying how location and dose of botulinum toxin injections affect muscle paralysis. Muscle Nerve 1993;16:964-9.

22. Childers MK, Kornegay JN, Aoki R, Otaviani L, Bogan DJ, Petroski G. Evaluating motor end-plate-targeted injections of botulinum toxin type A in a canine model. Muscle Nerve 1998;21:653-5.

23. Aquilonius SM, Askmark H, Gillberg PG, Nandedkar S, Olsson Y, Stalberg E. Topographical localization of motor endplates in cryosections of whole human muscles. Muscle Nerve 1984;7:287-93.

24. Christensen E. Topography of terminal motor innervation in striated muscles from stillborn infants. Am J Phys Med 1959;38:65-78.

25. Mu L, Sanders I. Human tongue neuroanatomy: nerve supply and motor endplates. Clin Anat 2010;23:77791.

26. Won SY, Rha DW, Kim HS, Jung SH, Park ES, Hu KS, et al. Intramuscular nerve distribution pattern of the adductor longus and gracilis muscles demonstrated with Sihler staining: guidance for botulinum toxin injection. Muscle Nerve 2012;46:80-5.

27. Ye JF, Lee JH, An XC, Lin CH, Yue B, Han SH. Anatomic localization of motor entry points and accurate regions for botulinum toxin injection in the flexor digitorum superficialis. Surg Radiol Anat 2011;33:601-7.

28. Lee JH, Lee BN, Han SH, An XC, Chung RH. The effec- 
tive zone of botulinum toxin A injections in the sternocleidomastoid muscle. Surg Radiol Anat 2011;33:18590.

29. Lee JH, Han SH, Ye JF, Lee BN, An X, Kwon SO. Effective zone of botulinum toxin a injections in hallux claw toe syndrome: an anatomical study. Muscle Nerve 2012;45:217-21.

30. Im S, Han SH, Choi JH, Lee JH, Ko YJ, Lee JI, et al. Anatomic localization of motor points for the neuromuscular blockade of hand intrinsic muscles involved in thumb-in-palm. Am J Phys Med Rehabil 2008;87:703-9.

31. Van Campenhout A, Molenaers G. Localization of the motor endplate zone in human skeletal muscles of the lower limb: anatomical guidelines for injection with botulinum toxin. Dev Med Child Neurol 2011;53:10819.

32. Oddy MJ, Brown C, Mistry R, Eastwood DM. Botulinum toxin injection site localization for the tibialis posterior muscle. J Pediatr Orthop B 2006;15:414-7.

33. Woodley SJ, Mercer SR. Hamstring muscles: architec- ture and innervation. Cells Tissues Organs 2005; 179:125-41.

34. Shaari CM, George E, Wu BL, Biller HF, Sanders I. Quantifying the spread of botulinum toxin through muscle fascia. Laryngoscope 1991;101:960-4.

35. Ma J, Smith BP, Smith TL, Walker FO, Rosencrance $\mathrm{EV}$, Koman LA. Juvenile and adult rat neuromuscular junctions: density, distribution, and morphology. Muscle Nerve 2002;26:804-9.

36. Amirali A, Mu L, Gracies JM, Simpson DM. Anatomical localization of motor endplate bands in the human biceps brachii. J Clin Neuromuscul Dis 2007;9:306-12.

37. Wu BL, Sanders I. A technique for demonstrating the nerve supply of whole larynges. Arch Otolaryngol Head Neck Surg 1992;118:822-7.

38. Drake W 3rd, Li Y, Rothschild MA, Wu BL, Biller HF, Sanders I. A technique for displaying the entire nerve branching pattern of a whole muscle: results in $10 \mathrm{ca}-$ nine posterior cricoarytenoid muscles. Laryngoscope 1993;103:141-8. 